\title{
Voltage Regulation Planning for Distribution Networks Using Multi-Scenario Three-Phase Optimal Power Flow
}

\author{
Antonio Rubens Baran Junior*(D), Thelma S. Piazza Fernandes and Ricardo Augusto Borba \\ Electrical Engineering Department, Federal University of Paraná (UFPR), Curitiba 81531-980, Brazil; \\ thelma@eletrica.ufpr.br (T.S.P.F.); borba6@gmail.com (R.A.B.) \\ * Correspondence: juniorbaran@gmail.com
}

Received: 29 October 2019; Accepted: 25 December 2019; Published: 29 December 2019

check for updates

\begin{abstract}
Active distribution networks must operate properly for different scenarios of load levels and distributed generation. An important operational requirement is to maintain the voltage profile within standard operating limits. To do this, this paper proposed a Multi-Scenario Three-Phase Optimal Power Flow (MTOPF) that plans the voltage regulation of unbalance and active distribution networks considering typical scenarios of operation. This MTOPF finds viable operation points by the optimal adjustments of voltage regulator taps and distribution transformer taps. The differentiating characteristic of this formulation is that in addition to the traditional tuning of voltage regulator taps of an active network applied for just one scenario of load and generation, it also performs the optimal adjustment of distribution transformer taps, which, once fixed, is able to meet the voltage limits of diverse operating situations. The optimization problem was solved by the primal-dual interior-point method and the formulation was tested using the IEEE 123-bus system.
\end{abstract}

Keywords: Three-phase optimal power flow; voltage regulation; distributed generation; distribution transformer taps

\section{Introduction}

The evolution of distribution networks affected their planning and operational philosophy by requiring load and distributed generation (DG) unbalances to be appropriately represented in the computational analysis tools. Thus, given the complexity of distribution networks, there is a tendency to avoid the simplified single-phase representation of the system in favor of the three-phase representation, which is more in keeping with the reality of $13.8-\mathrm{kV}$ and $34.5-\mathrm{kV}$ lines.

Many previous studies have explored traditional voltage regulation equipment as described by the authors of [1]: Step voltage regulators, switched capacitors, and on-load tap changer (OLTC).

As summarized by the authors of [1], there are advanced methods to realize the voltage regulation, such as generation curtailment during low demand, reactive power control by reactive compensator (VAR compensation), continuously changing the tap changer setting at substation, inverters of smart DG, consumption shifting and curtailing, energy storage, and microgrids providing ancillary service.

In Section 2, the voltage regulation equipment, mathematical formulations, and methods used to confront the challenges of planning and operation of active distribution networks are presented. In Section 2, it is shown that none of the actual studies have used these voltage regulation equipment and methods to make an optimal allocation of distribution transformers taps (DT), which is traditional equipment that can be a better adjustment to better integrate the advanced technologies.

So, before the allocation of these more sophisticated technologies, we proposed a proper selection of DT taps in a way to enjoy more benefits from these technologies, which are important to face the new challenges of voltage profile variations of active networks. 
Thus, this research adjusted a single-period Three-Phase Optimal Power Flow (TOPF), proposed by the authors of [1], into a multi-scenario formulation that assists the voltage regulation planning of a distribution network, making not only the traditional adjustments of voltage regulators, but also of the distribution transformers taps (DT) that simultaneously satisfy different configurations of load and DG power injections (that depends on the solar incidence, for example) during a typical day.

The adjustments proposed in this article considered a large insertion of photovoltaic (PV) generation, which required careful monitoring to not exceed operational limits of the network and equipment. Therefore, to address these questions, it was necessary to act on the step voltage regulators and on the distribution transformers taps that existed along the feeders to control the voltage profile.

To do this, the purpose of this article was to propose an optimization problem that applies to a three-phase unbalanced network (besides the conventional control actions, such as voltage regulator taps, as [2]) and DT tap adjustments to monitor the voltage profile, considering not only one point of operation, but a combination of multiple scenarios simultaneously (MTOPF). The consideration of multiple periods (or scenarios) must be made because after the DTs taps are fixed at planned positions, they do not change during the operation time. So, this tap allocation satisfies different conditions of load and DG penetrations (with pre-establishment of a different combination of scenarios) while minimizing the total electrical losses.

Besides the description of the MTOPF proposed, some variations of this main idea were developed in a way to validate its results. We also proposed a parametrization of loads and GD insertions that allowed the execution of each scenario individually. This formulation was named PTOPF, and it can confront the results of a single-period formulation with a multi-period formulation, showing the advantages of the multi-period proposed in this article. Besides these implementations, we also proposed a method that exhaustively tested all the combinations of DT taps to validate the results of the MTOPF and PTOPF.

The results showed that the formulations obtained a configuration of taps for all DTs while optimizing the steps of voltage regulators, finding viable points of operations along a typical day of an active distribution network. Additionally, the formulation found operational points that minimized the total electrical losses.

This work is organized in eight different sections. First, a brief bibliographic review on analysis models for three-phase networks is presented, followed by a description of the three-phase models used in the formulation proposed in this article. Next, the proposed MTOPF formulation is presented with the new considerations introduced to it, followed by a load parametrization modeled to make the validation of the distribution transformers taps optimized. Finally, the results and conclusions are presented for the IEEE 123-bus system.

\section{Literature Review}

Analytical tools available for the solution of three-phase load flows (LF) have already been developed, such as [3-9]. In addition, the authors of [10] described an open-source system simulator (OpenDSS) that has interface integration with other programs.

All these reported works described conventional LF solutions for three-phase distribution systems. However, more complex strategies to plan voltage control require more attention, especially when there are many single-phase loads connected from the main three-phase trunk, which causes the unbalanced operating voltage across the feeder, increasing losses and hampering voltage regulation, along with the three phases of the circuit.

The voltage control is also important when there are DGs installed at radial feeders, which can induce reverse power flows and change the voltage profile, requiring proper interventions because the original voltage regulation schemes may not meet the requirements after the DG access.

These challenges corroborate the importance of proposing mathematical formulations that consider the networks unbalanced and can make adjustments that are necessary to maintain the operational quality requirements, considering many different scenarios of load and power injection of DGs. 
These adjustments can be obtained through optimization problems, such as the Three-Phase Optimal Power Flow (TOPF). Some of them are resolved via the interior point method (IPM), such as:

- $\quad$ Reference [11], which presented a TOPF equated with four-wire current injection, which focused on voltage unbalance analysis and simulates the IEEE 34- and 123-bus systems;

- Reference [12], which analyzed the effects of single-phase and three-phase PV generation on losses and voltage profile; and

- $\quad$ Reference [3], which represented the balance equations through power injections to adjust voltage regulator taps and bank of capacitors.

There are other works that solve TOPF through other methods, such as quasi-Newton, semi-defined programming (SDP), or second-order cone programming, such as:

- $\quad$ Reference [13], which used the quasi-Newton method in conjunction with OpenDSS at Smart Grid applications to redraw the reactive power to reduce electrical losses; and

- $\quad$ References [14-17], which used SDP to solve TOPF when there was a large penetration of DG, focusing on the adjustment of voltage regulator taps.

Despite the advantages of SDP, such as finding an ideal global solution, it was not selected for this study due to the large computational effort required in the simulation of large systems, as it increases the search space of the solution by increasing the number of variables.

The previously described references are related to the mathematical formulation of three-phase systems and methods to solve them, emphasizing the relevance of the IPM, which was also used in this work to solve the optimization problem proposed.

Next, the equipment and methods used to control voltage regulation are cited.

As already mentioned, there are many works that explored traditional voltage regulation equipment, such as step voltage regulators [2], switched capacitors [1], and on-load tap changers (OLTC) [1]. The applications of more sophisticated technologies have also been described by previous works, including low-voltage static var compensators (LV-SVCs) [18], distributed energy-storage systems [19], static synchronous compensator (STATCOM) [20], and microgrids providing ancillary service [21].

But, none of these works optimized the taps using traditional equipment, such as the DTs, which are not yet sufficiently explored to better integrate with the advanced technologies. So, we proposed a proper optimization of the DT taps in a way to enjoy more benefits from them and from the advanced technologies, which are important to face the new challenges of voltage profile variations of active networks.

Some kinds of DT tap adjustments were already performed in [22], which used genetic algorithms to solve the optimizations problem, together with the OpenDSS, which analyzed each solution generated. The authors of [23] proposed a quadratic three-phase transformer model resolved via a mixed-integer quadratically constrained quadratic program model, binary scheme and big-M method.

However, these formulations only considered one operating point, and for different scenarios, they must be executed again, obtaining different DT taps allocations for each individual execution. However, these tap positions remain fixed throughout their operations, and they must simultaneously satisfy several scenarios that cover different load levels and PV generation (several solar radiation profiles), among other parameters that change throughout the day.

Thus, the tuning of DTs must be able to satisfy various operating scenarios of the network to achieve an allocation that meets all of them.

Recent works $([24,25])$ presented formulations that used a stochastic method to design high DG penetration in distribution networks, considering various operating scenarios. In [25], the scenarios considered wind generation, and in [24], PV generation was modeled. There is another strand of work that used neural networks [26], which require a long length of time to make the training of data.

Using numerical approaches to simultaneously address several questions about adjustments and three-phase representations, we proposed an improvement to the single-period TOPF modeled by [2] 
(which used the already well-established IPM), by the addition of DT tap adjustments (to bypass the high-voltage drop at the end of the feeder and avoid exceeding operating limits) and expanded the single-period performance to a multi-period conception that considers simultaneously the most representative scenarios of load levels and DG penetrations. So, the DT taps were allocated in a way to satisfy all the most significant load and DG conditions of a typical day.

This formulation was named Multi-Scenario Three-Phase Optimal Power Flow (MTOPF). It was solved via IPM technique, which was selected over other techniques because it was used to solve nonlinear and non-convex problems, such as an OPF, with good results.

To do it, we proposed a quadratic power injection model that represents the taps of distribution transformers together with a multi-period version of the TOPF [2] to make possible the consideration of many scenarios of load and solar incidence simultaneously. The objective was to find a tap position that was ideal throughout a typical day operation and that minimized the total electrical losses.

Thus, in this article, the MTOPF was proposed. Besides the traditional tuning of voltage regulator taps of an active three-phase network applied for just one period, the MTOPF also has the following functionalities and contributions:

- $\quad$ Resolve the nonlinear active and reactive power balance equations;

- Adjust the DT taps (considering the transformer winding connections) to adapt the voltage profile to many periods of operation; and

- $\quad$ Adjust the voltage regulator taps.

Besides the proposition of the MTOPF, the parametrization of loads and GD insertions was also proposed, which allowed a sequential resolution of each scenario individually. This formulation, named PTOPF, was used to confront the results between a single-period formulation and a multi-period formulation, and showed the advantages of the last one, which was the main differential of this work, as it encompasses the intertemporally of the problem. After these implementations a method was also proposed that exhaustively tested all the combinations of DT taps to validate the results of the MTOPF and PTOPF.

\section{Distribution Systems}

Distribution networks have predominantly unbalanced connections, radial topology, and different types of loads and lines [27].

In this work, the modeling line used was the circuit $\pi$ equivalent to a three-phase line, as proposed by the authors of [23], considering the mutual inductances between the phases. The three-phase admittance matrix, $\dot{\boldsymbol{Y}}_{b u s}^{A, B, C}$, was organized by blocks by phase because the power balance equations of the problem are also organized by phase $(\mathrm{A}, \mathrm{B}, \mathrm{C})$ [1]. The three-phase modeling loads used were connected in a star configuration, and their representations can be [28] active and reactive power constant, constant current, or constant impedance models, or any combination of the three models. Capacitors were modeled as a star ground configuration. The capacitive susceptance of a bank of capacitors connected at bus $i$, phase $p h$, can be obtained as:

$$
c_{i}^{p h}=\frac{Q_{i}^{p h}}{\left|\dot{V}_{i}^{p h}\right|^{2}}
$$

where $Q_{i}^{p h}$ is the nominal reactive power of the capacitors at bus $i$, phase $p h$, and $\left|\dot{V}_{i}^{p h}\right|$ is the voltage magnitude at bus $i$, phase $p h$.

The voltage regulators have an automatic control of taps to adjust the voltage network according to preset parameters. In this work, the voltage regulators were modeled as three single-phase units with a transformation ratio equal to $1: a$, where $a$ is the ratio of the magnitudes of the voltages. The 
transformation ratio $a$ affected the elements of the three-phase admittance bus matrix, as described by the authors of [2].

There are many different types of transformer windings. In this work, the wye-grounded/wye-grounded connection was modeled. The equivalent circuit for this kind of DT is presented by the authors of [29] (Figure 1). This DT has an off-nominal tap ratio, $\alpha: \beta$, between the primary and the secondary windings, where $\alpha$ and $\beta$ are the taps on the primary and secondary sides, respectively. In this work, $\beta$ was considered at the nominal position $(\beta=1)$ and $\alpha$ was conveniently adjusted. The admittance value of the transformer was $\dot{y}_{t}$.

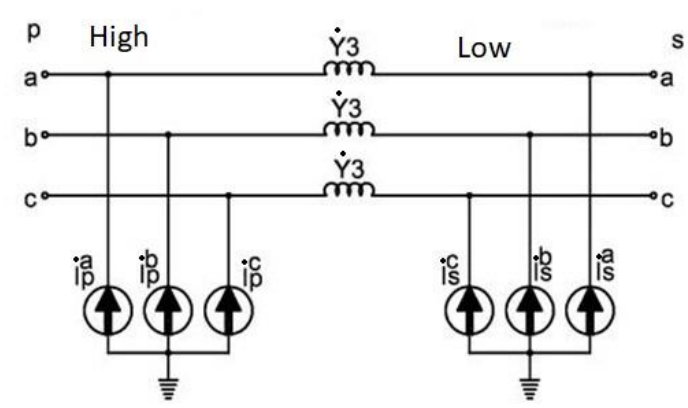

Figure 1. Representation of a distribution transformer with a wye-grounded/wye-grounded connection [28].

The value of $\dot{Y}_{3}$ and the current injections of Figure 1 are described as follows, in phasor form [28]:

$$
\begin{gathered}
\dot{Y}_{3}=\frac{\dot{y}_{t}}{\alpha \beta} \\
\bar{I}_{p}^{p h}=\frac{\dot{y}_{t}}{\alpha \beta}\left(\frac{\beta-\alpha}{\alpha}\right) \bar{V}_{p}^{p h}, \\
\bar{I}_{s}^{p h}=\frac{\dot{y}_{t}}{\alpha \beta}\left(\frac{\alpha-\beta}{\beta}\right) \bar{V}_{s}^{p h}
\end{gathered}
$$

where $\bar{I}_{p}^{p h}$ is the current injected at the primary of phase $p h, \bar{I}_{s}^{p h}$ is the current injected at the secondary of phase $p h, \bar{V}_{p}^{p h}$ is the voltage at the primary of phase $p h$, and $\bar{V}_{s}^{p h}$ is the voltage at the secondary phase $p h$.

Hence, the structure of the three-phase admittance bus matrix of the distribution transformer, $\dot{Y}_{\mathrm{D}}^{a b c}$, is like the three-phase admittance matrix of a three-phase line whose admittance is equal to $\dot{Y}_{3}$ without mutual coupling between phases.

It should be pointed out that the authors of [28] proposed this power injection representation to overcome singularity problems. This representation was also used in this work to adjust the taps $(\alpha)$ of the DTs using the MTOPF, which is described in the next section.

\section{Formulation of the Multi-Scenario Three-Phase Optimal Power Flow}

This section presents the mathematical formulation of the proposed multi-scenario three-phase optimal power flow. The objective function of the proposed method is the minimization of losses. As a differential, the method also has the adjustment of DT taps, considering $n p$ scenarios at the same time.

As described by the authors of $[9,30]$, due to the known advantageous properties of the rectangular form, instead using the polar form to represent the voltage phasor, this MTOPF uses the rectangular form.

So, the nodal phasor voltage, $\dot{V}^{p h, n p}$, is represented by the rectangular form as:

$$
\dot{V}^{p h, t}=e+j \cdot f, t=1, \ldots, n p, p h=1,2,3
$$


where $e$ is the real component of the phasor voltage with dimension (3.nb.np $\times 1), f$ is the imaginary component of the phasor voltage, $n b$ is the number of buses of the network, and $n p$ is the number of scenarios (or periods).

The real and imaginary components of the nodal phasor voltage are expressed as the vector $\mathbf{x}$ :

$$
\mathbf{x}=\left[\mathbf{e}^{T} f^{T}\right]^{T}
$$

where $\mathbf{x}$ has dimension $(6 . n b . n p \times 1)$.

The general formulation of the optimization problem is:

$$
\text { Min Losses }=\sum_{p h=1}^{3} \sum_{t=1}^{n p} \sum_{i=1}^{n b}\left(P g h_{i}^{p h, t}+P g d_{i}^{p h, t}-P d_{i}^{p h, t}\right)
$$

subject to

$$
\begin{aligned}
& \mathbf{P g}^{p h, t}+\mathbf{P g d}^{p h, t}-\mathbf{P d}^{p h, t}=\mathbf{P}^{p h, t}\left(x, a^{p h, t}, \alpha\right) \cdot x, \\
& \mathbf{Q g}^{p h, t}+\mathbf{Q g d} \mathbf{d}^{p h, t}+\operatorname{diag}\left(\left|\dot{V}^{p h, t}\right|^{2} \cdot c^{p h, t}-\mathbf{Q d}^{p h, t}=\mathbf{Q}^{p h, t}\left(x, a^{p h, t}, \alpha\right) \cdot \boldsymbol{x},\right. \\
& \boldsymbol{P g}_{\text {min }} \leq \boldsymbol{P g}^{\text {ph, }} \leq \boldsymbol{P} \boldsymbol{g}_{\text {max }} \\
& Q g_{\text {min }} \leq \mathbf{Q g}^{p h, t} \leq Q g_{\text {max }} \\
& V_{\text {min }}^{2} \leq\left|\dot{V}^{p h, t}\right|^{2} \leq V_{\max }^{2} \\
& \boldsymbol{a}_{\min } \leq \boldsymbol{a}^{p h, t} \leq \boldsymbol{a}_{\max }, \\
& \boldsymbol{\alpha}_{\min } \leq \boldsymbol{\alpha} \leq \boldsymbol{\alpha}_{\max },
\end{aligned}
$$

where Losses is the objective function that minimizes losses; $P g^{p h, t}$ is the vector of active power generation of each phase $p h$ and scenario $t$ with dimension (3.nb.np $\times 1) ; P d^{p h, t}$ is the vector of active power load of each phase $p h$ and scenario $t$ with dimension (3.nb.np $\times 1) ; Q g^{p h, t}$ is the vector of reactive power generation of each phase $p h$ and scenario $t$ with dimension (3.nb.np $\times 1) ; Q d^{p h, t}$ is the vector of reactive power load of each phase $p h$ and scenario $t$ with dimension (3.nb.np $\times 1) ; P g d^{p h, t}$ is the vector of active power generation of distributed generation of each phase $p h$ and scenario $t$ with dimension (3.nb.np $\times 1)$; $Q g d^{p h, t}$ is the vector of reactive power generation of distributed generation of each phase $p h$ and scenario $t$ with dimension $(3 . n b . n p \times 1) ; a^{p h, t}$ is the ratio voltage magnitudes of voltage regulators of each phase $p h$ and scenario $t$ with dimension (3.nreg.np $\times 1$ ); nreg is the number of voltage regulators; $\boldsymbol{a}_{\min }$ and $\boldsymbol{a}_{\max }$ are the minimum and maximum voltage magnitude ratio of the voltage regulators with dimension (3.nreg.np $\times 1$ ); $c^{p h, t}$ is the capacitive susceptance of capacitor banks installed at $n c$ buses with dimension (3.nb.np $\times 1$ ); $P g_{\min }$ and $P g_{\max }$ are the minimum and maximum limits of the active generation with dimension (3.nb.np $\times 1) ; Q g_{\text {min }}$ and $Q g_{\max }$ are the minimum and maximum limits of the reactive generation with dimension (3.nb.np $\times 1$ ); $V_{\min }$ and $V_{\max }$ are the minimum and maximum limits of voltage magnitude phasor with dimension (3.nb.np $\times 1)$; $\alpha$ is the vector of taps adjusted on the primary of DT with dimension $(n d t \times 1)$, where $n d t$ is the number of distributer transformers; and $\boldsymbol{\alpha}_{\text {min }}$ and $\boldsymbol{\alpha}_{\max }$ are the minimum and maximum tap position of the DTs with dimension $(n d t \times 1)$.

The functions $\mathbf{P}^{p h, t}\left(x, a^{p h, t}, \alpha\right) \cdot x$ and $\mathbf{Q}^{p h, t}\left(x, a^{p h, t}, \alpha\right) \cdot x$, which represent the active and reactive power injections of each bus, respectively, are quadratic equations due to the rectangular representation of the voltage phasor [31]. To illustrate this, the vector $\mathbf{P} \mathbf{d}^{p h, t}$ (active power load) and the vector $\mathbf{Q} \mathbf{d}^{p h, t}$ (reactive power load) have the following layout:

$$
\begin{aligned}
\mathbf{P d}^{p h, t}= & {\left[\begin{array}{lllllll}
P d_{1}^{a, 1} \ldots P d_{n b}^{a, 1} & P d_{1}^{b, 1} \ldots P d_{n b}^{b, 1} P d_{1}^{c, 1} \ldots P d_{n b}^{c, 1} \ldots P d_{1}^{a, n p} \\
& \ldots P d_{n b}^{a, n p} P d_{1}^{b, n p} \ldots P d_{n b}^{b, n p} P d_{1}^{c, n p} \ldots P d_{n b}^{c, n p}
\end{array}\right]^{T}, }
\end{aligned}
$$




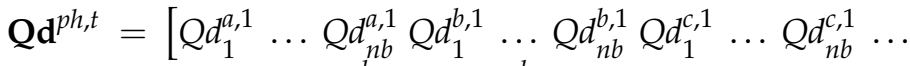

$$
\begin{aligned}
& \left.Q d_{1}^{a, n p} \ldots Q d_{n b}^{a, n p} Q d_{1}^{b, n p} \ldots Q d_{n b}^{b, n p} Q d_{1}^{c, n p} \ldots Q d_{n b}^{c, n p}\right]^{T} \text {, } \\
& t=1, \ldots, n p
\end{aligned}
$$

where $\mathrm{Pd}_{\mathrm{i}}^{\mathrm{k}, \mathrm{t}}$ represents the active power load at bus $i$, phase $k$, and scenario $t$, and $\mathrm{Qd}_{\mathrm{i}}^{\mathrm{k}, \mathrm{t}}$ represents the reactive power load at bus $i$, phase $k$, and scenario $t$.

The vector of nodal complex voltage has the following layout:

$$
\begin{aligned}
& \dot{\boldsymbol{V}}^{p h, t}(\mathbf{x})=\left[\begin{array}{llllllllll}
\dot{V}_{1}^{a, 1} & \ldots & \dot{V}_{n b}^{a, 1} \dot{V}_{1}^{b, 1} & \ldots & \dot{V}_{n b}^{b, 1} \dot{V}_{1}^{c, 1} & \ldots & \dot{V}_{n b}^{c, 1} & \ldots
\end{array}\right. \\
& \left.\dot{V}_{1}^{a, n p} \ldots \dot{V}_{n b}^{a, n p} \dot{V}_{1}^{b, n p} \ldots \dot{V}_{n b}^{b, n p} \dot{V}_{1}^{c, n p} \ldots \dot{V}_{n b}^{c, n p}\right]^{T}, \\
& t=1, \ldots, n p \text {. }
\end{aligned}
$$

The optimization variables of the MTOPF are as follows: The vector $\mathbf{x}$ represents the nodal voltage phasor modeled by the rectangular form $\left(\dot{V}^{p h, t}(\mathbf{x})\right), \mathbf{P g}^{p h, t}$ represents the active power generation, $\mathbf{Q g}^{\text {ph,t }}$ represents the reactive power generation, $a^{p h, t}$ represents the taps of the voltage regulator, and $\alpha$ represents the taps of DTs.

The limits of voltage magnitude values are squared because the voltage phasor is represented in the rectangular form [30].

The multi-scenario three-phase optimal power flow formulated from Equations (7)-(14) is solved by the primal-dual interior-point method. This method obtains the best solution, keeping the search inside the area delimited for restrictions. So, it changes the inequalities into equations of equality, through the introduction of slack variables. The main characteristic of this numerical method is the addition of a logarithmic barrier function to the objective function to guarantee the non-negativity of the slack variables. In sequence, the Karush-Kuhn-Tucker (KKT) conditions that express the first optimality conditions of the optimization problem are resolved by the application of Newton's method to obtain the solution of the non-linear equations (KKT). This method was selected due to its good performance obtained to solve traditional OPF [31,32] of real systems.

\subsection{Three-Phase Distribution Transformer Model}

The three-phase distribution transformer model can be done in two ways [28]: Using the admittance bus matrix or injecting current into the primary and secondary buses of the transformer as shown in Figure 1.

The distribution transformer model that inserts the taps directly into current injection equations (Equations (2)-(4)) circumvents problems of numerical conditioning in relation to the proposal that inserts the taps directly into the bus admittance matrix [28].

In addition, this strategy facilitates the derivation of the first and second derivatives that must be calculated to optimize the taps $(\alpha)$ of the DTs via the IPM.

Since the active and reactive power balance equations of the optimization problem were modeled using power injections (as described by the authors of [2]), the current injections of the DT were transformed into power injections:

$$
\begin{array}{ll}
\dot{S}_{p}^{p h, t}=\dot{V}_{p}^{p h, t}\left[\frac{\dot{y}_{3}}{\alpha}\left(\frac{1-\alpha}{\alpha}\right) \dot{V}_{p}^{p h, t}\right]^{*} & t=1, \ldots, n p, p h=1, \ldots, 3 \\
\dot{S}_{s}^{p h, t}=\dot{V}_{s}^{p h, t}\left[\frac{\dot{y}_{3}}{\alpha}\left(\frac{\alpha-1}{1}\right) \dot{V}_{s}^{p h, t}\right] & t=1, \ldots, n p, p h=1, \ldots, 3
\end{array}
$$

The equations of power injections (18) can be represented as

$$
\dot{\boldsymbol{S}}_{p}=\operatorname{diag}\left(\dot{\boldsymbol{V}}^{p h, t}\right)\left[\frac{\dot{y}_{3}}{\alpha}\left(\frac{1-\alpha}{\alpha}\right) \cdot \operatorname{Inc} Y Y p \cdot \dot{\boldsymbol{V}}^{p h, t}{ }^{*} \quad t=1, \ldots, n p, p h=1, \ldots, 3\right.
$$


where the vector $\dot{S}_{p}$ represents the power injections at the primary buses of the DT, and IncYYp is a matrix of zeros with dimension (3.nb.np $\times$ 3.nb.np). The diagonal positions of $I n c Y Y p$ that correspond to the primary transformer buses were assumed to have values equal to 1 .

The equations of power injections (19) can be represented as:

$$
\dot{S}_{s}=\operatorname{diag}\left(\dot{\boldsymbol{V}}^{p h, t}\right)\left[\frac{\dot{y}_{3}}{\alpha}\left(\frac{\alpha-1}{1}\right) \cdot \operatorname{Inc} Y Y_{s} \cdot \dot{V}^{p h, t^{*}}\right] \quad t=1, \ldots, n p, p h=1, \ldots, 3
$$

where $\dot{\mathbf{S}}_{s}$ represents the power injections at the secondary buses of the DT, and Inc YYs is a matrix of zeros with dimension $(3 . n b . n p \times 3 . n b . n p)$. The diagonal positions of $I n c Y Y s$ that correspond to the secondary transformer buses were assumed to have values equal to 1 .

The power injections $\dot{S}_{p}$ and $\dot{S}_{s}$ make up the power balance equations related to the DTs, $S_{\text {trafo }}$ :

$$
S_{\text {trafo }}=\dot{S_{p}}+\dot{S_{s}}
$$

which are quadratic equations because the nodal voltages are represented in a rectangular form via the vector $x$.

So, the complete power balance equations of the network are:

$$
\begin{gathered}
\mathbf{P g}{ }^{p h, t}+\operatorname{Pg} d^{p h, t}-\mathbf{P d}^{p h, t}=\mathbf{P}^{p h, t}\left(x, a^{p h, t}, \alpha\right) \cdot x+\operatorname{real}\left[S_{\text {trafo }}\left(x, a^{p h, t}, \alpha\right) \cdot x\right] \cdot \\
\mathbf{Q g}^{p h, t}+Q g d^{p h, t}+\operatorname{diag}\left(\left|\dot{V}^{p h, t}\right|^{2} \cdot \cdot^{p h, t}-\mathbf{Q d}^{p h, t}=\mathbf{Q}^{p h, t}\left(x, a^{p h, t}, \alpha\right)+S_{\text {trafo }}\left(x, a^{p h, t}, \alpha\right) \cdot x\right]
\end{gathered}
$$

The great advantage of this multi-scenario formulation is its ability to obtain a configuration of DT taps which satisfies all operational constraints not only for a given period, but for a range of possible scenarios that can occur.

Normally, a DT has five taps with $2.5 \%$ steps. Therefore, the steps that can be selected are 1 , $0.975,0.95,0.925$, and 0.9 . So, as the MTOPF formulated provides continuous tap values, they must be discretized. The discretization technique used in this work was based on the proposal formulated in [28] to operate capacitor banks.

So, the continuous values $\left(\propto_{i}\right)$ need to be discretized $\left(\propto_{\text {disc } \_}\right)$, which can assume two values: A tap position immediately above the continuous tap $\left(\operatorname{Tap}_{\min \_} i\right)$ or a tap position immediately below the continuous tap $\left(\operatorname{Tap}_{\max _{-} i}\right)$, where $\propto_{\text {disc } \_i}$ is selected as the value closest to the continuous tap $\left(\propto_{i}\right)$ :

$$
\propto_{\text {disc } \_i}=\min \left(\left[\left(\propto_{i}-\operatorname{Tap}_{\min \_} i\right),\left(\operatorname{Tap}_{\max \_} i-\propto_{i}\right)\right]\right) .
$$

\section{Load and Solar Incidence Parametrization}

The adjustments of the DT taps were also calculated using a parameterized TOPF, which followed the evolution of load and solar incidence of a typical day.

The optimization problem formed by Equations (7)-(14) was adapted in a way that $n p=1$ (named as TOPF), which was repeatedly and separately simulated for only one scenario that corresponds to each moment of a day, obtained by a homotopy function.

The homotopy function allowed the variation of load and solar incidence over $n h=24 \mathrm{~h}$ ( $n h$ is the number of hours) by the variation of the index $\varepsilon(\varepsilon=0, \ldots, n h)$.

The proposed homotopy function was:

$$
\begin{gathered}
\boldsymbol{P} d^{p h}=\boldsymbol{P} d_{\varepsilon=0}^{p h}+\Delta \boldsymbol{P} d_{\varepsilon}^{p h} \\
Q d^{p h}=Q d_{\varepsilon=0}^{p h}+\Delta Q d_{\varepsilon}^{p h} \\
P g d^{p h}=P \operatorname{Pg} d_{\varepsilon=0}^{p h}+\Delta P g d_{\varepsilon}^{p h} \quad \varepsilon=0, \ldots, \mathrm{nh}
\end{gathered}
$$


where each $\Delta \boldsymbol{P} d_{\varepsilon}^{p h}, \Delta Q d_{\varepsilon}^{p h}$, and $\Delta \boldsymbol{P} g d_{\varepsilon}^{p h}$ were values that provide load and active distribution generation adjustments over a given period of $n h$ hours. If the period of operation is a day, the value of $n h$ is $24 \mathrm{~h}$ (or any other interval of interest).

The TOPF runs sequentially $n h$ times, following the parameterization of load and PV generation. For each period $\varepsilon$ analyzed, the tap values of each $\mathrm{DT}_{i}, \alpha_{\varepsilon}^{i}$, were optimized together with the other optimization variables.

After the parameterization process, different DT taps were obtained for each instant differently from what happens from the MOPT results, which provide a unique tap for each DT and meet all the scenarios simultaneously.

So, a unique tap position must be found from all the taps calculated from the parametrization process. The discretization is made like [33].

So, to discretize the continuous taps, $\alpha_{\varepsilon}^{i}$, of each $\mathrm{DT}_{i}$ and each $\varepsilon$, the following equation was used, assuming a study horizon of $n h$ hours:

$$
\begin{gathered}
\alpha_{\min }^{i}=\min \left(\alpha_{\varepsilon}^{i}\right) \\
\alpha_{\text {disc } \_i}=\min \left(\left[\left(a_{\text {min }}^{i}-\operatorname{Tap}_{\min \_i}\right)\left(\operatorname{Tap}_{\max \_i}-a_{\text {min }}^{i}\right)\right]\right)
\end{gathered}
$$

After the achievement of each $\propto_{\text {disc } \_i}$, they were fixed, and the TOPF was calculated again $n h$ times to simulate and check if there were no violations of the voltage limits with the taps adjusted.

\section{Results}

The objective of this section was to present results related to the proposed methodology. The distribution systems used were the IEEE 123-bus (Figure 2), adapted by the inclusion of four voltage regulators (VR) with all loads connected in wye-grounded configuration with constant power representation and two further DTs included: The first between the buses 44 and 47 (DT 1) and the second between the buses 89 and 91 (DT 2). Both distribution transformers had the same impedance parameters as the already connected three-phase transformer between the buses 61 and 124 (DT 3).

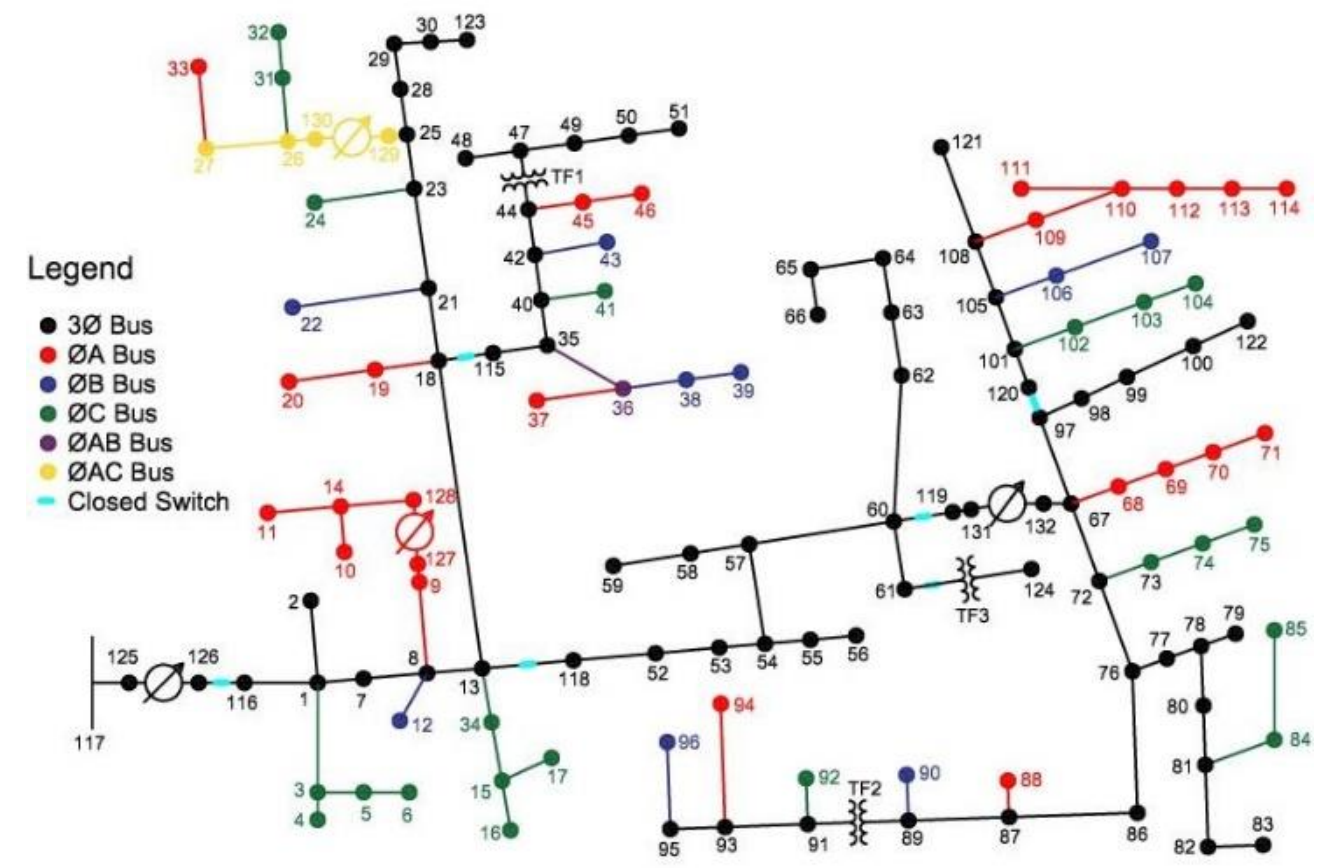

Figure 2. IEEE 123-Bus System (adapted). 
Nine distributed equivalent solar generators were also added, which were allocated at buses 47 , $48,49,50,51,91,93,95$, and 124 .

Another change from the original system was the addition of loads to increase the voltage drop in the system, and consequently, force different nominal tap adjustments.

The adopted base was $5 \mathrm{MVA}, 4.16 \mathrm{kV}$ with a tolerance of $1 \times 10^{-4}$. Nine scenarios were considered, which represent different load configurations and Photovoltaic (PV) generation, as shown in Table 1.

Table 1. Composition of the scenarios, load and PV generation percentage for each period.

\begin{tabular}{|c|c|c|c|c|}
\hline & $\% \mathrm{PV}$ & 0 & 52.84 & 96.15 \\
\hline \%Load & & \multicolumn{3}{|c|}{ Scenario } \\
\hline 81.10 & \multirow{3}{*}{ 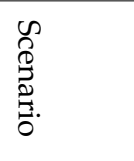 } & 1 & 2 & 3 \\
\hline 95.90 & & 4 & 5 & 6 \\
\hline 110.70 & & 7 & 8 & 9 \\
\hline
\end{tabular}

The value $\% \mathrm{PV}$ is the percentage of power generated by nominal PV power and depends on the solar irradiation levels. The value \%Load is the percentage of original power load in a way to represent different load levels. In addition, different PV penetration percentages were also considered: $0 \%, 20 \%$, and $70 \%$ in relation to the nominal heavy load. These penetration values are in relation to the $\% \mathrm{PV}$ percentage.

According to Table 1, there were three load levels $(81.10 \%, 95.90 \%$, and $110.70 \%)$ and three insolation factors $(0 \%, 52.84 \%$, and $96.15 \%)$, which were repeated three times for each load level. For example, for scenarios 1, 2, and 3, the load level was $81.10 \%$ with insolation factors of $0 \%, 52.84 \%$, and $96.16 \%$, respectively.

In addition, as the values of DT taps' ( $\alpha)$ are continuous variables and the tap positions are discrete values, they must be discretized (as described in Section 4.1). After the discretization, the Multi-Scenario Three-Phase Optimal Flow is simulated again, but with fixed discrete taps to verify the feasibility of the results.

In the sequence, the adjusted taps are presented to $0 \%, 20 \%$, and $70 \%$ of PV penetration using the MTOPF (Sections 6.1-6.3).

To obtain these PV values for different penetration levels, loads from scenarios 7, 8, and 9 were used, which were the largest system loads (heavy load), represented in Figure 3. The corresponding PV generations for a level of $20 \%$ and $70 \%$ penetration are shown in Figures 4 and 5, respectively. No penetration of $0 \%$ is shown because there is no PV generation in this condition.

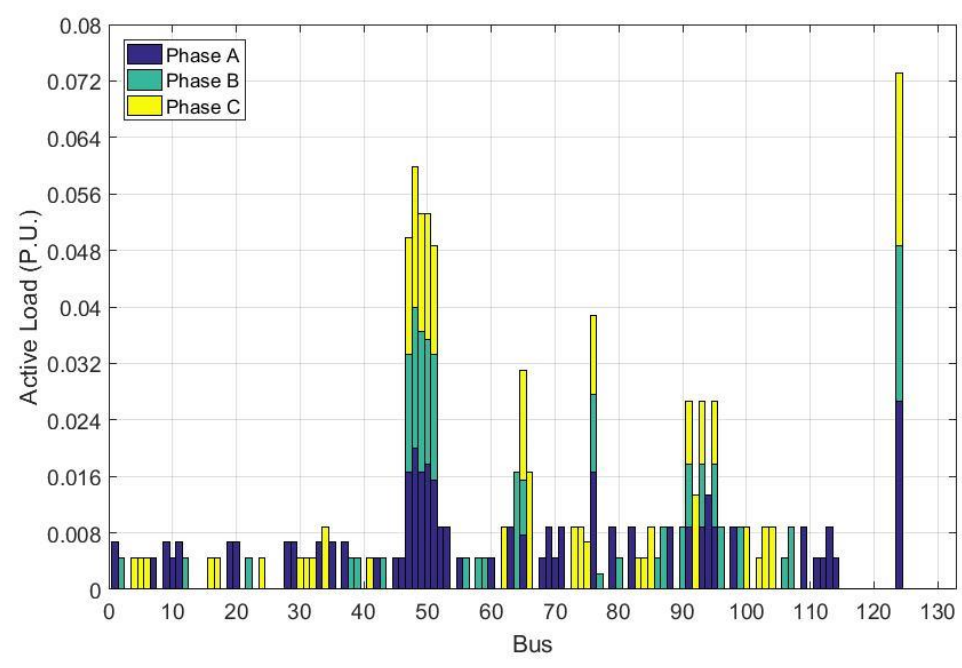

Figure 3. IEEE 123-Bus System Load (adapted). 


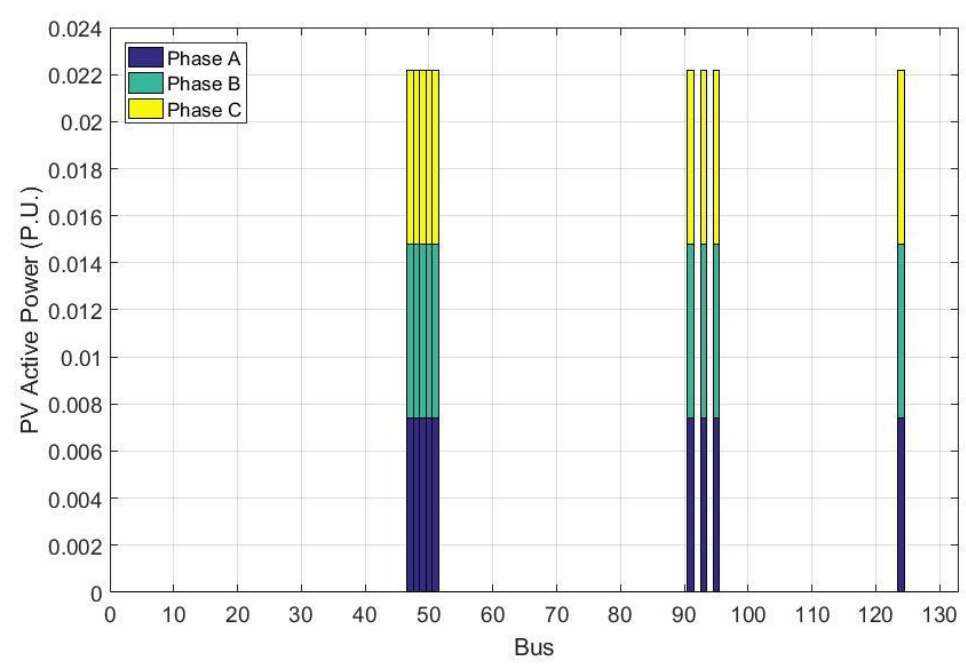

Figure 4. IEEE 123-Bus Maximum PV Active Power, 20\% PV Penetration.

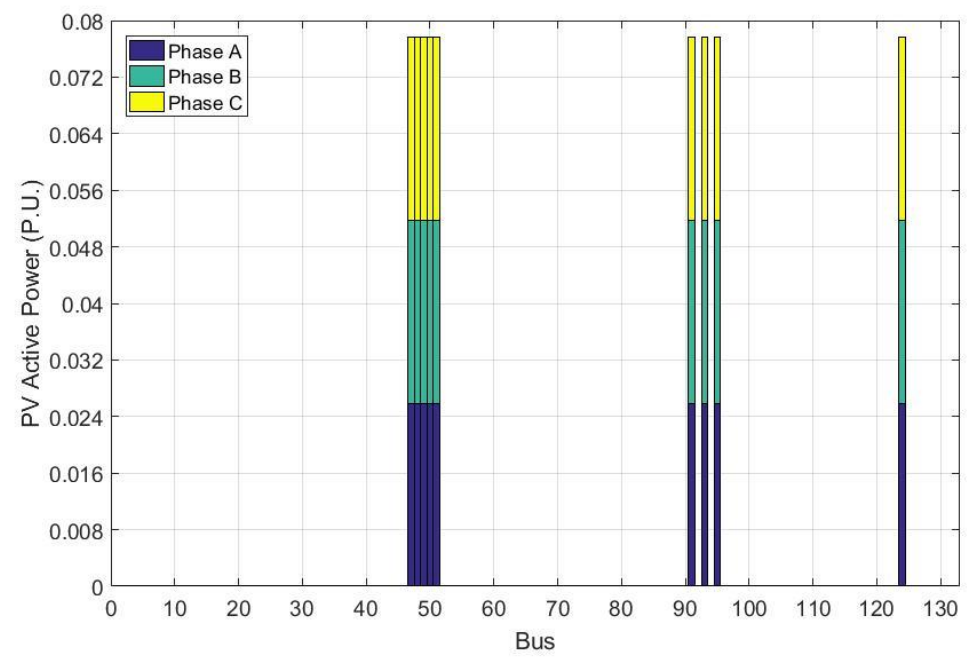

Figure 5. IEEE 123-Bus Maximum PV Active Power, 70\% PV Penetration.

The total system of heavy load condition was 0.9985 p.u., with distributions at 0.3941 P.U. in phase A, 0.2756 p.u. in phase B, and 0.3288 p.u. in phase C. The PV generation of the system for $0 \%$ penetration was 0 p.u.. For $20 \%$ penetration the total PV generation was 0.1997 p.u., being 0.0666 p.u. for each phase. For a $70 \%$ penetration the total PV generation was 0.6990 p.u., being 0.2330 p.u. for each of the phases.

In Section 6.4, the results obtained with the tap adjustments by the MTOPF are compared with one simulation fixing all taps at nominal value. This simulation was done to analyze the impacts of the MTOPF results.

In Section 6.5, the results of one exhaustive method, tests all the possible combinations of tap positions of the three distribution transformers of IEEE-123-bus, ae presented. This was done with the purpose of validating the MTOPF results.

In Section 6.6, the results of the Parameterized Three-Phase Optimal Power Flow (PTOPF) are presented and compared with the MTOPF.

All tests were performed on a computer operating with Windows 10 system, with the following configuration: Intel I5-8400 processor, Gigabyte B360M AORUS G3 motherboard, memory g.skill 2x8gb $2400 \mathrm{Mhz}$ (working in dual channel mode), Samsung SSD 500GB 850EVO sata3, VGA Sapphire Radeon R9 270X toxic 2GB. 
The convergence of the optimization problem formulated via the interior point method depends on the adjustment of some intrinsic parameters of the method, such as the initial barrier parameter, duality gap acceleration factor, and variable initialization. Once these parameters are well-adjusted, the computational performance depends on the characteristics of the computer, such as floating-point operations per second (flops) of each CPU core and enough memory to encompass all the code and data.

\subsection{Results Using 123-Bus System 0\% of PV Penetration}

For a configuration without generation penetration, according to Table 1, only three scenarios, referring to three load levels, were analyzed.

The continuous and discrete DT taps adjusted are shown in Figure 6.

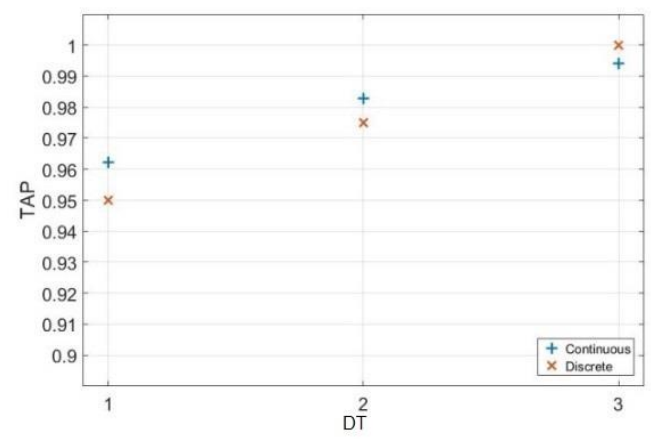

Figure 6. Continuous and discrete adjustment of taps, $0 \% \mathrm{PV}$.

Since the results of the voltage magnitudes are similar for each phase, the results are only presented for phase A. Figures 7 and 8 show the voltage magnitudes of phase A using continuous and discrete taps, respectively. All voltage magnitude values were within the established limits.

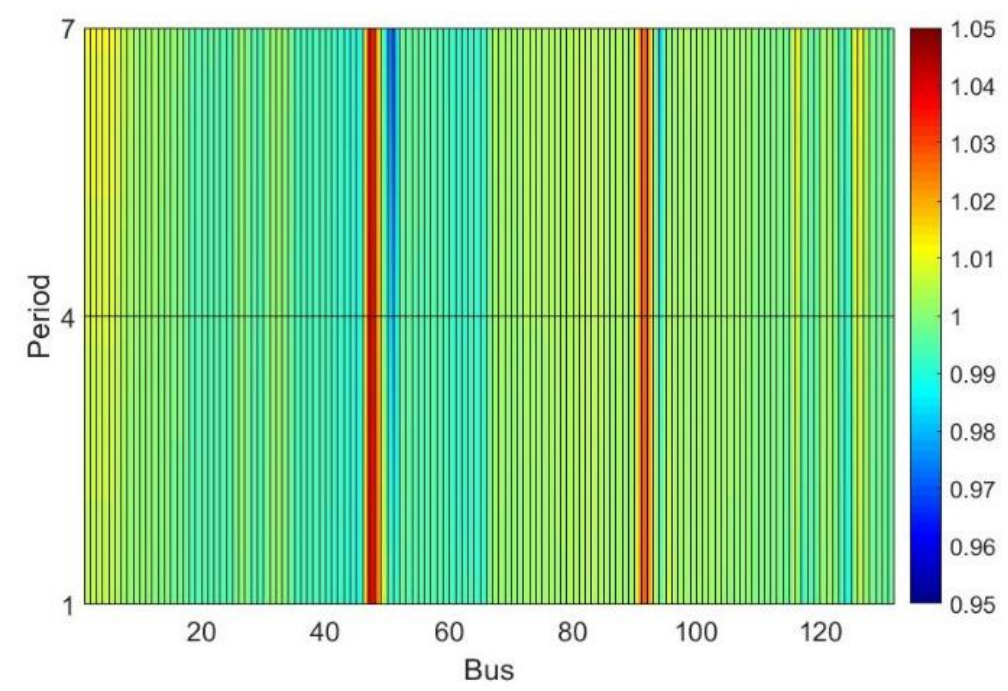

Figure 7. IEEE 123-bus system, voltage magnitudes of phase A, discrete taps, $0 \% \mathrm{PV}$.

All graphs of voltage magnitude (Figure 7, Figure 8, Figure 10, Figure 11, Figures 13-15) use a pseudo-color device to enable the representation of a wide range of data generated. $X$-axis values represent the buses of the system, $Y$-axis bars represent the periods (or scenarios), and the colors of each coordinate $(x, y)$ are defined by the matrix of voltage magnitude values of each bus and each period. The color of each segment depends on the values assumed at each of its four vertices. The color corresponds to the value of the first vertex, and there is an interpolation between the vertexes of each segment to smooth the color representation. 


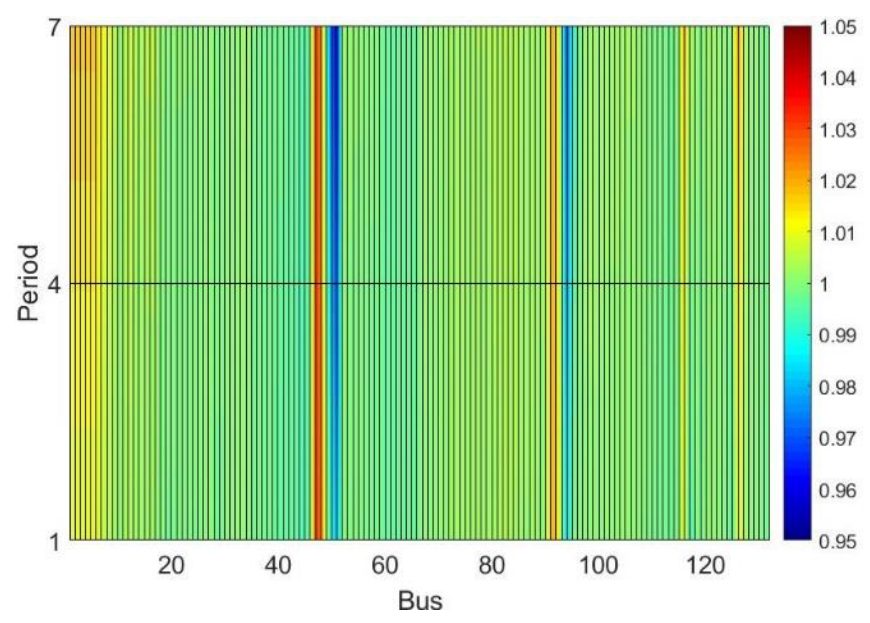

Figure 8. IEEE 123-bus system, voltage magnitudes of phase A, continuous taps, $0 \% \mathrm{PV}$.

As the values found in the continuous and discrete modes were not the same, but similar, it is observed, as expected, that the values of voltage magnitude were also slightly different between the two adjustment modes. This was the case except for buses 47 and 91, which showed larger variations because they were precisely at secondary buses of DT 1 and 2 that were adjusted outside the nominal position.

As a result of tap adjustments, the magnitude voltage after the secondary buses of the DTs was higher than the voltage at the primary, thus contributing to an increase in the voltage profile of the buses installed downstream of the DTs. Note that some buses had their voltages reduced, as some buses had high voltages. This was partly due to the voltage regulators, which had their taps adjusted differently in each case.

\subsection{Results Using 123-Bus System $20 \%$ of PV Penetration}

For the configuration with $20 \%$ of PV penetration and according to Table 1, nine scenarios were considered.

The taps adjusted in continuous and discrete modes are shown in Figure 9, while Figures 10 and 11 show the voltage magnitudes of phase A using continuous and discrete modes, respectively. All voltage magnitude values were within the established limits.

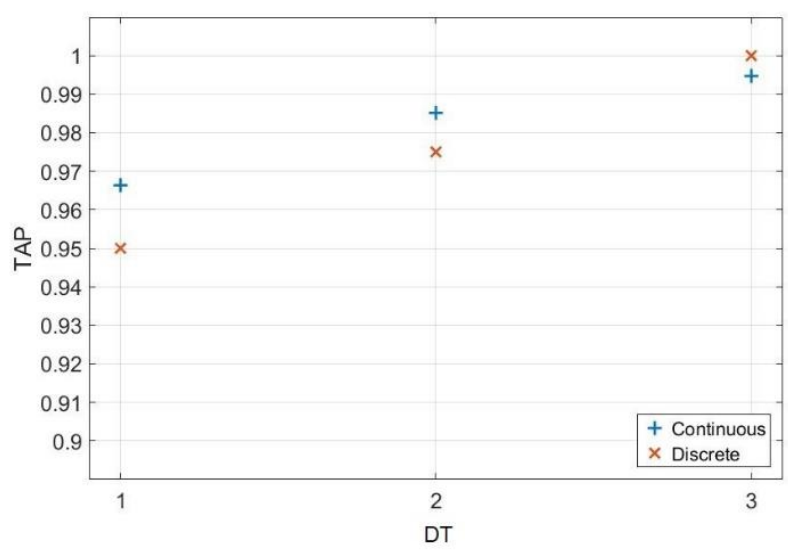

Figure 9. Continuous and discrete adjustment of taps, 20\% PV. 


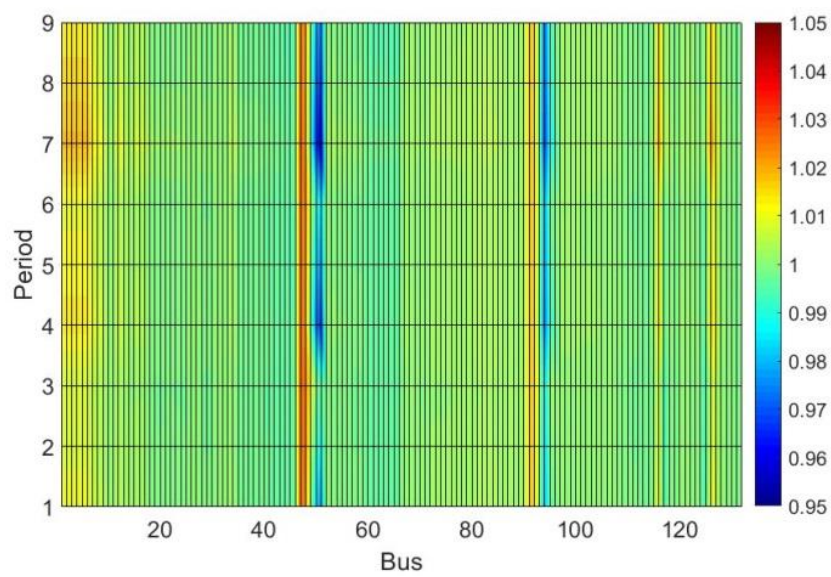

Figure 10. IEEE 123-bus system, voltage magnitudes of phase A, continuous taps, $20 \% \mathrm{PV}$.

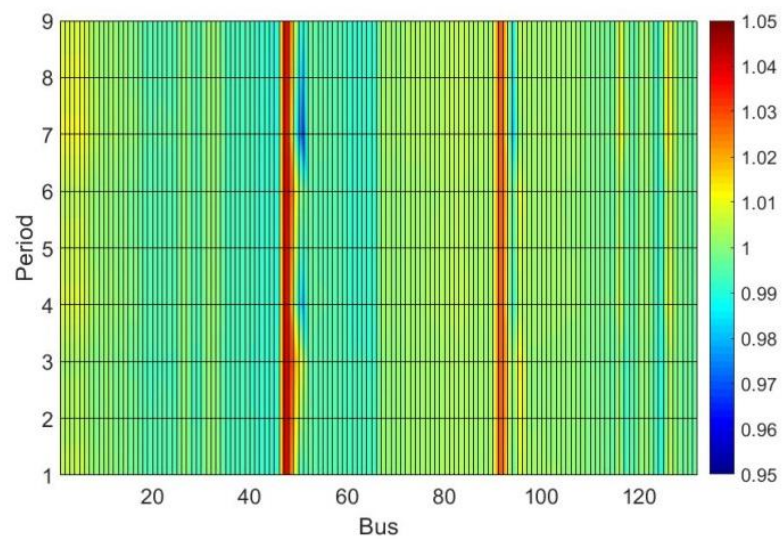

Figure 11. IEEE 123-bus system, voltage magnitudes of phase A, discrete taps, 20\% PV.

For example, observing the adjustment of DT 1 in Figure 9, it can be observed that its optimal continuous tap value was approximately equal to 0.966 P.U. Since the discrete taps were set with 0.025 P.U. steps, the discrete tap closest to the optimal continuous value would be 0.975 P.U. However, the discrete value was adjusted to the value equal to 0.95 P.U., which was adequate to keep the voltage magnitude within the operational limits as presented in Figure 10, which shows the voltage magnitudes of phase A. Continuous tap values of period 7 at buses 50 and 51 (secondary downstream buses of DT1) were represented by blue tones very close to the value of 0.95 P.U. If the tap of DT 1 was set to 0.975 P.U., this voltage magnitude would be further reduced, thus violating the minimum voltage limit. Another important fact is that at bus 47, which corresponded to the secondary bus of DT 1 , the voltage magnitude was represented by a red tone close to 1.03 P.U., which made it possible to reduce the value of the discrete tap, which caused an increase in voltage.

The increase in voltage profile generated by the discretization of taps can be seen in Figure 11. At bus 41, the color became a stronger red color, indicating that the voltage magnitudes were closer to 1.05 P.U.. The voltage magnitudes at bus 51 for each period had a significant increase, and during period 7, it was a lighter shade of blue than shown in Figure 10.

Another important difference between Figures 10 and 11 is that there was a reduction in the magnitude voltage at several buses. In Figure 10, there are many points where the voltage magnitudes are represented by the colors green and yellow (1 to 1.02 P.U.). In Figure 10, the same buses are represented by the colors cyan and green (0.98 to 1 P.U.).

Even with the inclusion of PV generation, the taps were adjusted with the same discrete adjustments calculated in the case without PV generation. However, the voltage variations at the buses where the DTs were connected were more significant than in the previous case because the discretization imposed a greater deviation from the optimum continuous value. 
Another important point to note is the inclusion of PVs. At scenarios when they were activated (2, 5 , and 8 with intermediate generation values and at scenarios 3, 6, and 9 with maximum generation values), the voltage at the buses where the PVs were connected tended to increase the voltage profile, especially with light and medium load.

\subsection{Results Using 123-Bus System $70 \%$ of PV Penetration}

For the configuration with $70 \%$ of PV penetration and according to Table 1, nine scenarios were considered.

The taps adjusted in continuous and discrete modes are shown in Figure 12, while Figures 13 and 14 show the voltage magnitudes of phase A using continuous and discrete modes, respectively. All voltage magnitude values were within the established limits.

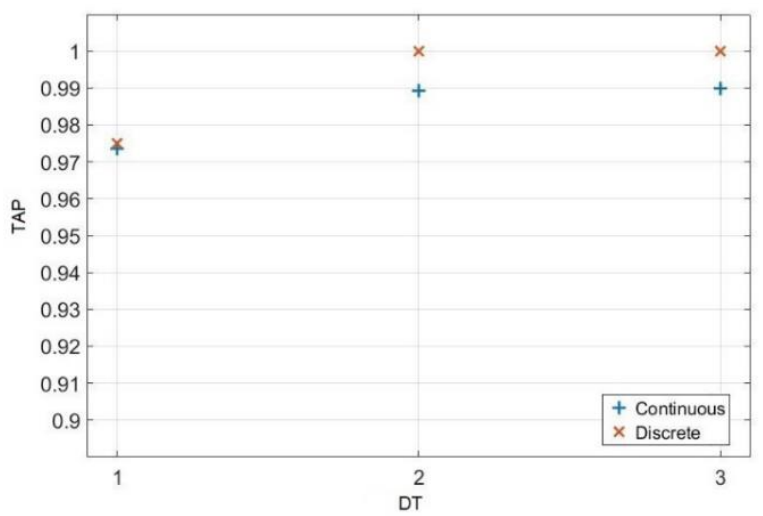

Figure 12. Continuous and discrete adjustment of taps, 70\% PV.

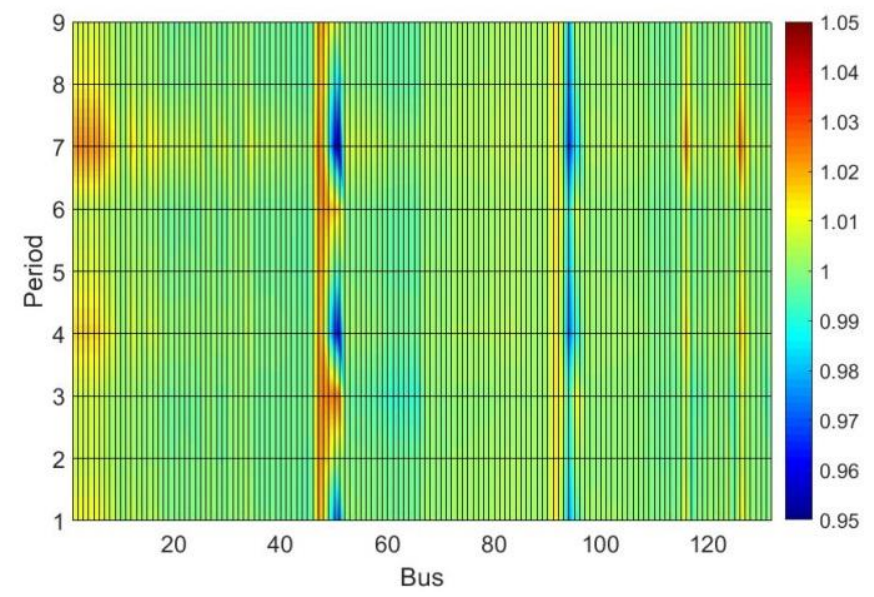

Figure 13. IEEE 123-bus system, voltage magnitudes of phase A, continuous taps, 70\% PV.

Analyzing Figure 12, it can be observed that the discrete tap adjustments were close to the continuous values. However, all discrete taps were set to values above the continuous. This implies a reduction of the voltage profile at secondary buses of the DTs, as can be seen when comparing Figures 13 and 14. In Figure 14, buses 50 and 51 (DT 1 secondary) and buses 93 and 96 (DT 2 secondary) have colors tending to a darker blue, indicating lower values, especially in period 7 (with heavy load and without PV generation).

Similar to the case with $20 \%$ PV, during the scenarios when PV was activated $(2,5$, and 8 with intermediate generation values and scenarios 3, 6, and 9 with maximum generation values), the magnitude of the voltage at buses where PVs were connected increased, especially with light and medium load. 
Through the results, the methodology allowed the adjustment of the DT taps, which were equipment already installed in the grid or that will be part of any grid expansion project, to control the voltage profile throughout active network systems, postponing installation of other equipment that, in many cases, may be costly to the utility (such as voltage regulators and static VAR compensators, among others).

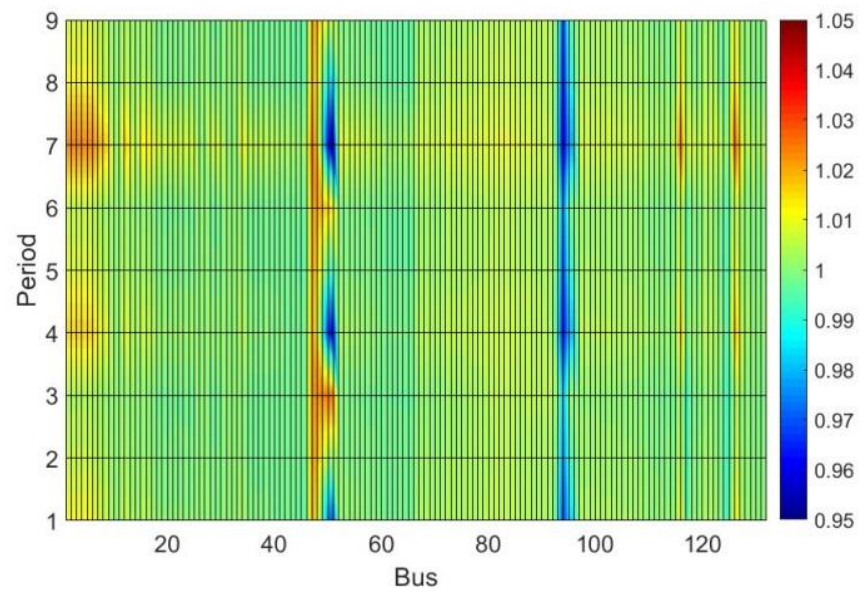

Figure 14. IEEE 123-bus system, voltage magnitudes of phase A, discrete taps, $70 \%$ PV.

Analyzing the calculated tap adjustments for different DG penetrations, it is observed for this system that when DG penetration increased, the tap positions tended toward the nominal position. This result was dependent on load level and penetration because as DG was distributed along the feeder, this fact decreased the current supplied by the substation and generally caused smaller voltage drops along the feeder. Thus, the tap adjustments tended to the nominal position to maintain voltages within the operating limits of the network.

\subsection{Adjusted TAP vs. Nominal TAP}

To show the impact at the voltage profile caused by the tap adjustments obtained by the proposed methodology, a simulation with $0 \% \mathrm{PV}$ and all taps fixed at the nominal value was performed.

The voltage profile in phase A obtained with the mentioned test is shown in Figure 15. Comparing Figure 15 with Figure 7 (where the taps were adjusted), the increase of the voltage profile was clear, with variations around 0.04 P.U.

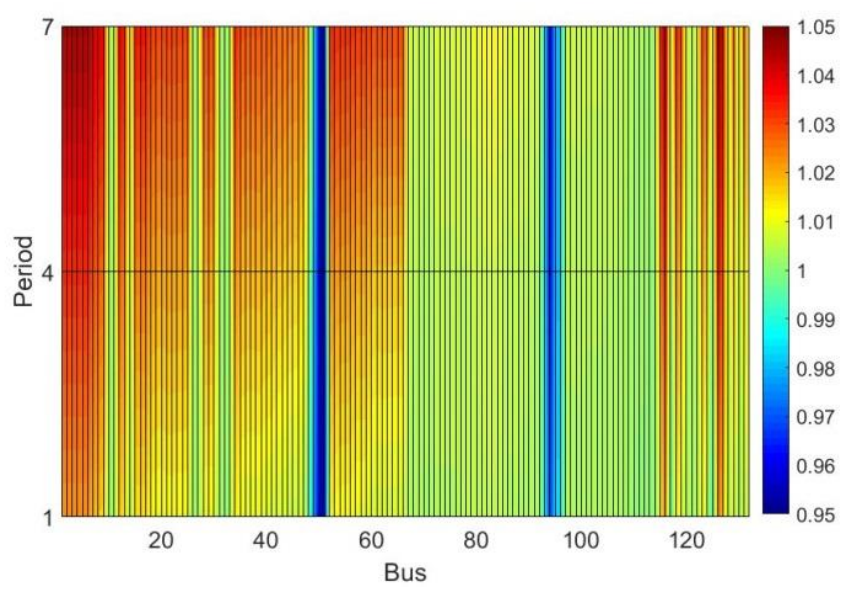

Figure 15. IEEE 123-bus system, voltage magnitudes of phase A, nominal taps, $0 \% \mathrm{PV}$. 
The voltage magnitude was not the only parameter that was impacted by the optimal adjustment of the taps. The electrical losses were also modified significantly. For example, for scenarios 1, 2, and 3, the losses were reduced by about $40 \%$ when the taps were optimized, as shown in Figure 16.

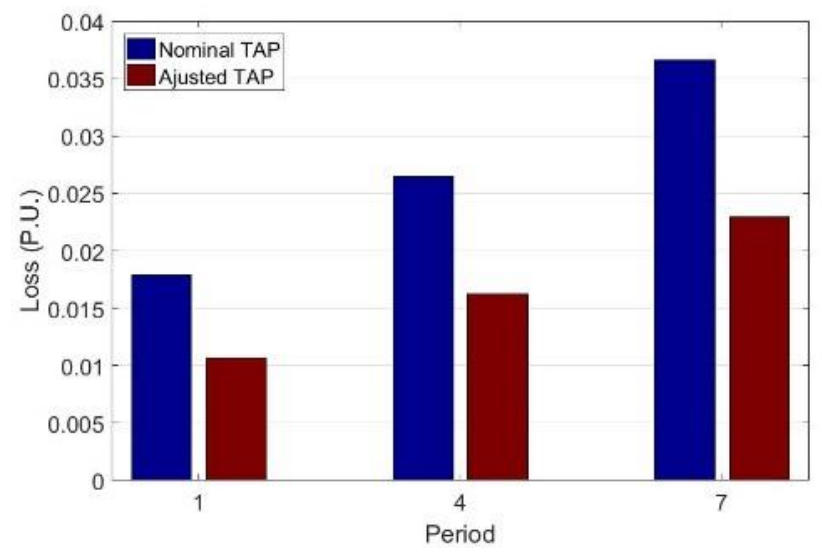

Figure 16. IEEE 123-bus system, electrical losses, $0 \%$ PV, with adjusted taps and nominal taps.

\subsection{Validation of Multi-Scenario Three-Phase Optimal Flow}

There is great difficulty in validating the results obtained through the Multi-Scenario Three-Phase Optimal Flow because there are no commercially available programs that perform the same functions as the proposed method.

One of the possibilities for the validation was to perform all possible combinations of tap positions of the three distribution transformers. So, considering that each DT had five positions available, the complete number of possible combinations of tap positions was 125.

To evaluate which combination of tap positions was the best, four parameters were analyzed: Total electrical loss, tendency to maintain voltage magnitudes around 1 P.U. (sum of voltage deviations from 1 P.U.), and the maximum and the minimum voltage deviations from 1 P.U.

The validation was executed using $0 \%$ of PV penetration and the scenarios of Table 1 .

After 125 simulations, 85 did not converge, 27 converged with loss values above the losses obtained by the optimal solution provided by the proposed methodology (optimal solution), and 13 converged with losses slightly below the losses obtained by the optimal solution.

Table 2 shows the 13 best candidate solutions and the solution with taps at nominal values. The optimal solution obtained by the optimization problem (MTOPF) was combination 8 .

Table 2. The best combination of tap positions.

\begin{tabular}{cccccc}
\hline Combination & $\begin{array}{c}\text { Taps of DTs } \\
(\mathbf{1}-\mathbf{2 - 3 )}\end{array}$ & $\begin{array}{c}\text { Loss } \\
\text { [P.U.] }\end{array}$ & $\begin{array}{c}\text { Sum: }|\boldsymbol{\Delta V}| \\
{[\text { P.U.] }}\end{array}$ & $\begin{array}{c}\Delta \mathbf{V} \text { Max } \\
{[\text { [.U.] }}\end{array}$ & $\begin{array}{c}\Delta \mathbf{V} \text { Min } \\
{[\text { P.U.] }}\end{array}$ \\
\hline 1 & $1-1-1$ & 0.24299 & 45.0706 & 0.0491 & -0.0498 \\
4 & $0.925-1-1$ & 0.135569 & 51.2721 & 0.0447 & -0.0448 \\
8 & $0.95-0.975-1$ & 0.149675 & 20.816 & 0.0466 & -0.0369 \\
9 & $0.925-0.975-1$ & 0.072057 & 54.5962 & 0.0497 & -0.0337 \\
28 & $0.95-1-0.975$ & 0.098848 & 24.6425 & 0.0481 & -0.0481 \\
29 & $0.925-1-0.975$ & 0.055553 & 58.1011 & 0.0498 & -0.0416 \\
33 & $0.95-0.975-0.975$ & 0.142254 & 19.8933 & 0.0463 & -0.037 \\
34 & $0.925-0.975-0.975$ & 0.08372 & 22.0082 & 0.0497 & -0.0339 \\
53 & $0.95-1-0.95$ & 0.115922 & 26.2772 & 0.0481 & -0.048 \\
54 & $0.925-1-0.95$ & 0.134602 & 51.223 & 0.0493 & -0.0449 \\
58 & $0.95-0.975-0.95$ & 0.135811 & 24.1333 & 0.0479 & -0.0402 \\
59 & $0.925-0.975-0.95$ & 0.150734 & 47.1414 & 0.0493 & -0.0341 \\
79 & $0.925-1-0.925$ & 0.07205 & 57.774 & 0.0498 & -0.0466 \\
84 & $0.925-0.975-0.925$ & 0.10125 & 53.1383 & 0.0497 & -0.0347 \\
\hline
\end{tabular}


First, analyzing the solution with the taps at the nominal position, it is observed that the losses were much higher than the other candidate solutions and the sum of the voltage deviations modulus in relation to 1 P.U. was among the largest, as well as the maximum and minimum voltage deviations.

Analyzing the best combinations obtained, it is possible to observe that for each analysis parameter, there were distinct optimal solutions. For example, according to the losses, the best solution was combination 29 , but the sum of the modulus of voltage deviations was the largest value among the simulations presented. Therefore, the analysis of the optimal solution also considered the incidence of higher maximum and minimum deviations.

The simulations that stand out when the four parameters were analyzed were combinations $\phi=\{8$, $28,33,53$, and 58\}. For example, the simulation with the lowest value of the electrical loss of the set $\phi$ was combination 28 , the one that presented the smallest sum of the voltage deviation modulus was combination 33 , the smallest variation of maximum $\Delta \mathrm{V}$ was combination 33 , and the smallest variation of minimum $\Delta \mathrm{V}$ was combination 8 .

Observing this set of discrete-tap solutions, the combination with the smallest $\Delta \mathrm{V}$ and the smallest sum of the voltage deviation was combination 33, but the proposed methodology indicated that combination 8 was the best solution. The difference between simulations 8 and 33, considering the maximum and minimum $\Delta \mathrm{V}$, was $0.03 \%$ and $0.01 \%$, respectively. The sum of the modulus of voltage deviations was $4.43 \%$, and the loss difference was $4.96 \%$. So, the two solutions were very close. These results can be more easily visualized when all the parameters are summed, which is presented in Table 3. So, the combination 33 was $0.33 \%$ better than solution 8 .

Table 3. Best adjustment of tap positions.

\begin{tabular}{ccc}
\hline Combination & $\begin{array}{c}\text { Taps of DTs } \\
(\mathbf{1}-\mathbf{2}-\mathbf{3})\end{array}$ & Sum \\
\hline 8 (continuous) & $0.9622-0.9828-0.9941$ & 14.1116 \\
8 (discrete) & $0.95-0.975-1$ & 21.049175 \\
33 & $0.95-0.975-0.975$ & 21.118854 \\
\hline
\end{tabular}

Comparing the values obtained by the optimal continuous solution, also presented in Table 3, the fitness of the solution was $33 \%$ better than the discrete solution. Then, the discretization caused slight distortion in the final result. However, it is an insignificant price to pay compared to executing all possible combinations.

For the nine scenarios considered, the MTOPF expended $3167 \mathrm{~s}$ to obtain the convergence. For each combination using the DT taps fixed, the MTOPF spent around $135 \mathrm{~s}$ (only for the cases that had feasible solutions). As previously described, 85 combinations of taps were not viable (in these cases, the simulations end after exceeding the number of iterations). So, to finish the 125 combinations of taps, the exhaustive method spent about $10 \mathrm{~h}$ in the total.

This combinatorial solution technique is completely unfeasible for larger systems. For example, if the network has $20 \mathrm{DTs}$, then 95 trillion combinations will be required to obtain the best configuration of taps. So, the advantage of the proposed method is that in a unique simulation, it obtains a viable solution that satisfactory assists a range of probable load and generation throughout the entire operation time.

To execute larger systems, the feeder can be divided into equivalent zones as proposed by the authors of [34]. Then, the MTOPF can be applied in equivalent areas.

Besides the application of exhaustive combination, which consumes a lot of time, the uses of other solution techniques, such as parametric processes, artificial intelligence technique, or mixed-integer nonlinear programming technique, are also costly.

Even so, the parameterized three-phase optimal power flow, presented in Section 5, was also executed in order to reinforce the results obtained by the proposed method, MTOPF, as presented in Section 6.5. 


\subsection{Parameterized Three-Phase Optimal Power Flow}

Unlike the MTOPF, which solved multiple scenarios at the same time, the parameterized TOPF solved multiple periods individually and separately, yielding a result for each period.

It can be emphasized that, as the discretization processes of each method are different (as described in Sections 4 and 5), they also led to slightly different adjustment results of DT taps, as shown in Table 4 . Table 4 presents the results obtained by parameterization (PTOPF), discrete MOPOF, and exhaustive simulations.

Table 4. Best continuous adjustment of tap positions.

\begin{tabular}{ccc}
\hline Method & $\begin{array}{c}\text { TAPs of DTs } \\
(\mathbf{1 - 2}-\mathbf{3})\end{array}$ & $\begin{array}{c}\text { Sum } \\
\text { [P.U.] }\end{array}$ \\
\hline PTOPF (discrete) & $0.975-1-1$ & 33.8697 \\
MTOPF (discrete) & $0.95-0.975-1$ & 21.9409 \\
Exhaustive Combinations & $0.95-0.975-0.975$ & 21.118854 \\
\hline
\end{tabular}

According to Table 4, each method presented different, but adherent, tap settings. Consequently, each method also presented different, but very close, total losses. These results validated the three strategies described to plan the allocation of distribution transformers taps that must support different levels of loads and levels of GD insertion.

The lowest loss values were obtained by the MTOPF (21.9409 P.U.) and by the exhaustive combinations (21.118854 P.U.). Although the result obtained by the exhaustive method was slightly better, the computational time spent by it was impeditive (around $10 \mathrm{~h}$ ).

The difference in adjustments between PTOPF and MTOPF occurred because PTOPF simulated each scenario individually and then adjusted the appropriate taps. That is, each scenario had its taps individually optimized only for that period, so this approach could not see the temporal connections between the periods, and its combinations results were not the best.

On the other hand, the MTOPF simulated all the scenarios at the same time, adjusting a unique combination of taps that satisfy all of them. So, each DT tap was adjusted to obtain the best positions for all periods considered simultaneously. Because of that, MTOPF is the method that provides the best results with good computational performance because it comprises a systematic process that encompasses several operating conditions simultaneously and executes the program only one time and not repeated for each individual scenario.

\section{Conclusions}

This article proposed an optimization problem, which was then applied to a three-phase unbalanced network. Besides the conventional control actions, such as voltage regulator taps, the optimization problem also optimizes DT taps to monitor the voltage profile considering not only one point of operation, but multiple combinations of load and photovoltaic generation, simultaneously (MTOPF).

The consideration of multiple periods (or scenarios) must be made, as after the DTs taps are fixed at planned positions, they do not change during the operation time. So, this tap allocation must satisfy a different combination of scenarios.

The MTOPF, which minimizes the total electrical loss, is solved by the primal-dual interior-point method. This method can be applied to planning studies to obtain the fixed adjustment of the taps of distribution transformers before of the application of more advanced methods and technologies useful to face the new challenges of voltage profile variations of active networks.

Some variations of the MTOPF were also developed in a way to validate its results as the parametrization of loads and GD insertions, which allowed the execution of each scenario individually (PTOPF). This formulation confronts the results of a single-period formulation (TOPF) with a multi-period formulation, showing the advantages of the multi-period. 
One of these advantages is the calculation of an optimal DT tuning plan that meets all load conditions and levels of generation during the operation time.

Another advantage is the computational time that MTOPF requires to obtain a solution, which is much faster and more efficient than the parameterized method (PTOPF).

Besides these implementations, a method that exhaustively tests all the combinations of DT taps was also proposed. The method successfully validated the results of the MTOPF and PTOPF.

Author Contributions: A.R.B.J. is responsible for the conception and implementation of the methodology, the generation of results was made by R.A.B. and T.S.P.F. coordinated the activities and reviewed the work. All authors have read and agreed to the published version of the manuscript.

Funding: Araucária Foundation and CAPES (Higher Education Personnel Improvement Coordination - Brazil): 88882.168617/2018-01

Acknowledgments: The authors are grateful for the support for this research provided by CNPq (National Council for Scientific and Technological Development - Brazil), CAPES (Higher Education Personnel Improvement Coordination - Brazil) and Araucária Foundation.

Conflicts of Interest: The authors declare no conflict of interest.

\section{References}

1. Mahmud, N.; Zahedi, A. Review of control strategies for voltage regulation of the smart distribution network with high penetration of renewable distributed generation. Renew. Sustain. Energy Rev. 2016, 64, 582-595. [CrossRef]

2. Baran, A.R.; Fernandes, T.S.P. A three-phase optimal power flow applied to the planning of unbalanced distribution networks. Int. J. Electr. Power Energy Syst. 2016, 74, 301-309. [CrossRef]

3. Cheng, C.S.; Shirmohammadi, D. A three-phase power flow method for real-time distribution system analysis. IEEE Trans. Power Syst. 1995, 10, 671-679. [CrossRef]

4. Shirmohammadi, D.; Hong, H.W.; Semlyen, A.; Luo, G.X. A compensation-based power flow method for weakly meshed distribution and transmission networks. IEEE Trans. Power Syst. 1988, 3, 753-762. [CrossRef]

5. Broadwater, R.P.; Chandrasekaran, A.; Huddleston, C.T.; Khan, A.H. Power flow analysis of unbalanced multiphase radial distribution systems. Electr. Power Syst. Res. 1988, 14, 23-33. [CrossRef]

6. Garcia, P.A.N.; Pereira, J.L.R.; Carneiro, S.; da Costa, V.M.; Martins, N. Three-phase power flow calculations using the current injection method. IEEE Trans. Power Syst. 2000, 15, 508-514. [CrossRef]

7. Teng, J.-H. A direct approach for distribution system load flow solutions. IEEE Trans. Power Deliv. 2003, 18, 882-887. [CrossRef]

8. Ramos, E.R.; Exposito, A.G.; Cordero, G.A. Quasi-Coupled Three-Phase Radial Load Flow. IEEE Trans. Power Syst. 2004, 19, 776-781. [CrossRef]

9. Penido, D.R.R.; de Araujo, L.R.; Carneiro, S.; Pereira, J.L.R.; Garcia, P.A.N. Three-Phase Power Flow Based on Four-Conductor Current Injection Method for Unbalanced Distribution Networks. IEEE Trans. Power Syst. 2008, 23, 494-503. [CrossRef]

10. Dugan, R.C. Reference Guide-The Open Distribution System Simulator (OpenDSS). Electric Power Researsch Institute, 2013. Available online: https://spinengenharia.com.br/wp-content/uploads/2019/01/ OpenDSSManual.pdf (accessed on 28 December 2019).

11. Araujo, L.R.; Penido, D.R.R.; Carneiro, S.; Pereira, J.L.R. A Three-Phase Optimal Power-Flow Algorithm to Mitigate Voltage Unbalance. IEEE Trans. Power Deliv. 2013, 28, 2394-2402. [CrossRef]

12. Ayikpa, M.E. Unbalanced distribution optimal power flow to minimize losses with distributed photovoltaic plants. Int. J. Electr. Comput. Energ. Electron. Commun. Eng. 2017, 11, 181-186.

13. Bruno, S.; Lamonaca, S.; Rotondo, G.; Stecchi, U.; La Scala, M. Unbalanced Three-Phase Optimal Power Flow for Smart Grids. IEEE Trans. Ind. Electron. 2011, 58, 4504-4513. [CrossRef]

14. Liu, Y.; Li, J.; Wu, L.; Ortmeyer, T. Chordal Relaxation Based ACOPF for Unbalanced Distribution Systems With DERs and Voltage Regulation Devices. IEEE Trans. Power Syst. 2018, 33, 970-984. [CrossRef]

15. Dall'Anese, E.; Zhu, H.; Giannakis, G.B. Distributed Optimal Power Flow for Smart Microgrids. IEEE Trans. Smart Grid 2013, 4, 1464-1475. [CrossRef] 
16. Bazrafshan, M.; Gatsis, N.; Zhu, H. Optimal Tap Selection of Step-Voltage Regulators in Multi-Phase Distribution Networks. In Proceedings of the 2018 Power Systems Computation Conference (PSCC), Dublin, Ireland, 11-15 June 2017; IEEE: Dublin, Ireland, 2018; pp. 1-7.

17. Robbins, B.A.; Zhu, H.; Dominguez-Garcia, A.D. Optimal Tap Setting of Voltage Regulation Transformers in Unbalanced Distribution Systems. IEEE Trans. Power Syst. 2016, 31, 256-267.

18. Padullaparti, H.V.; Nguyen, Q.; Santoso, S. Optimal Placement and Dispatch of LV-SVCs to Improve Distribution Circuit Performance. IEEE Trans. Power Syst. 2019, 34, 2892-2900. [CrossRef]

19. Wang, Y.; Tan, K.T.; Peng, X.Y.; So, P.L. Coordinated Control of Distributed EnergyStorage Systems for Voltage Regulation in Distribution Networks. IEEE Trans. Power Deliv. 2016, 31, 1132-1141.

20. Rohouna, W.; Balog, R.; Peerzada, A.A.; Begovi, M.M. D-STATCOM for harmonic mitigation in low voltage distribution network with high penetration on nonlinear loads. Renew. Energy 2020, 145, 1449-1464. [CrossRef]

21. Wang, X.; Wang, C.; Xu, T.; Guo, L.; Li, P.; Yu, L.; Meng, H. Optimal voltage regulation for distribution networks with multi-microgrids. Appl. Energy 2018, 210, 1027-1036. [CrossRef]

22. Vitor, T.S.; Vieira, J.C.M. Optimal voltage regulation in distribution systems with unbalanced loads and distributed generation. In Proceedings of the 2016 IEEE Innovative Smart Grid Technologies-Asia (ISGT-Asia), Melbourne, Australia, 28 November 2015-1 December 2016; IEEE: Melbourne, Australia, 2016; pp. 942-947.

23. Ju, Y.; Wu, W.; Lin, Y.; Ge, F.; Ye, L. Three-phase optimal load flow model and algorithm for active distribution networks. In Proceedings of the 2017 IEEE Power \& Energy Society General Meeting, Chicago, IL, USA, 16-20 July 2017; IEEE: Chicago, IL, USA, 2017; pp. 1-5.

24. Xu, J.; Wang, J.; Liao, S.; Sun, Y.; Ke, D.; Li, X.; Liu, J.; Jiang, Y.; Wei, C.; Tang, B. Stochastic multi-objective optimization of photovoltaics integrated three-phase distribution network based on dynamic scenarios. Appl. Energy 2018, 231, 985-996. [CrossRef]

25. Galdi, V.; Vaccaro, A.; Vallacci, D. Voltage regulation in MV networks with dispersed generations by a neural-based multiobjective methodology. Electr. Power Syst. Res. 2008, 78, 785-793. [CrossRef]

26. Mokryani, G.; Hu, Y.F.; Pillai, P.; Rajamani, H.-S. Active distribution networks planning with high penetration of wind power. Renew. Energy 2017, 104, 40-49. [CrossRef]

27. Chen, T.-H.; Chen, M.-S.; Hwang, K.-J.; Kotas, P.; Chebli, E.A. Distribution system power flow analysis-a rigid approach. IEEE Trans. Power Deliv. 1991, 6, 1146-1152. [CrossRef]

28. Stagg, G.W.; El-ablad, A. Computer Methods in Power System Analysis; McGraw-Hill Book Co.: New York, NY, USA, 1968; ISBN 978-0-07-060658-6.

29. Chen, M.-S.; Dillon, W.E. Power system modeling. Proc. IEEE 1974, 62, 901-915. [CrossRef]

30. Torres, G.L.; Quintana, V.H. An interior-point method for nonlinear optimal power flow using voltage rectangular coordinates. IEEE Trans. Power Syst. 1998, 13, 1211-1218. [CrossRef]

31. Fernandes, T.S.P.; Almeida, K.C. A methodology for optimal power dispatch under a pool-bilateral market. IEEE Trans. Power Syst. 2003, 18, 182-190. [CrossRef]

32. Wright, S.J. Primal-Dual Interior-Point Methods; Society for Industrial and Applied Mathematics: Philadelphia, PA, USA, 1997; ISBN 978-0-89871-382-4.

33. Dahlke, D.B.; Steilein, G.; Fernandes, T.S.P.; Aoki, A.R. A heuristic to adjust automatic capacitors using parameterization of load. Int. J. Electr. Power Energy Syst. 2012, 42, 16-23. [CrossRef]

34. Zhou, X.; Liu, Z.; Zhao, C.; Chen, L. Accelerated Voltage Regulation in Multi-Phase Distribution Networks Based on Hierarchical Distributed Algorithm. Math. Optim. Control 2019, 1903, 00072. [CrossRef]

(C) 2019 by the authors. Licensee MDPI, Basel, Switzerland. This article is an open access article distributed under the terms and conditions of the Creative Commons Attribution (CC BY) license (http://creativecommons.org/licenses/by/4.0/). 